INPLASY

PROTOCOL

To cite: Ji et al. Effect of early versus late tracheostomy in ventilated COVID-19 patients: a systematic review and metaanalysis. Inplasy protocol 202180088. doi:

10.37766/inplasy2021.8.0088

Received: 23 August 2021

Published: 23 August 2021

Corresponding author:

Yun Ji

yunji@zju.edu.cn

Author Affiliation:

Department of Surgical Intensive Care Unit, the Second Affiliated Hospital, School of Medicine, Zhejiang University.

Support: None.

Review Stage at time of this submission: Preliminary searches.

Conflicts of interest:

None declared.

\section{Effect of early versus late tracheostomy in ventilated COVID-19 patients: a systematic review and meta-analysis}

Ji, Y1; Fang, Y2; Li, L³.

Review question / Objective: Is early tracheostomy superior to late tracheostomy in ventilated COVID-19 patients for the following clinical outcomes (i) duration of mechanical ventilation, (ii) duration of ICU stay, (iii) mortality.

Condition being studied: Tracheostomy is one of the most common procedures in ventilated COVID-19 patients. Whether early tracheostomy reduces mortality and duration of mechanical ventilation and ICU stay is controversial. The aims of our study are to review existing clinical evidence and evaluate the impact of early tracheostomy compared to late tracheostomy in ventilated COVID-19 patients.

INPLASY registration number: This protocol was registered with the International Platform of Registered Systematic Review and Meta-Analysis Protocols (INPLASY) on 23 August 2021 and was last updated on 23 August 2021 (registration number INPLASY202180088).

\section{INTRODUCTION}

Review question / Objective: Is early tracheostomy superior to late tracheostomy in ventilated COVID-19 patients for the following clinical outcomes (i) duration of mechanical ventilation, (ii) duration of ICU stay, (iii) mortality.

Condition being studied: Tracheostomy is one of the most common procedures in ventilated COVID-19 patients. Whether early tracheostomy reduces mortality and 
duration of mechanical ventilation and ICU stay is controversial. The aims of our study are to review existing clinical evidence and evaluate the impact of early tracheostomy compared to late tracheostomy in ventilated COVID-19 patients.

\section{METHODS}

Participant or population: COVID-19 patients who require mechanical ventilation undergoing tracheostomy.

Intervention: Early tracheostomy (as defined by the included studies) in ventilated COVID-19 patients.

Comparator: Late tracheostomy (as defined by the included studies) in ventilated COVID-19 patients.

Study designs to be included: We will include retrospective or prospective observational studies and randomized controlled trials.

Eligibility criteria: Studies will be included if they compare outcomes of early and late tracheostomy in ventilated COVID-19 patients. Studies not providing necessary outcomes data will be excluded.

Information sources: Systematic literature search will be performed in Medline, Embase, Cochrane, and Scopus database.

Main outcome(s): Duration of mechanical ventilation, duration of ICU stay, and mortality.

Quality assessment / Risk of bias analysis: We will assess risk of bias using the Cochrane Handbook for Systematic Reviews of Interventions.

Strategy of data synthesis: The estimation of combined continuous values and dichotomous values will be expressed as mean differences (MD) or odds ratios (OR), respectively, with $95 \%$ confidence intervals (CI). An $12>50 \%$ will be considered indicative of significant heterogeneity.
Sensitivity analysis: None.

Country(ies) involved: China.

Keywords: COVID-19, Mechanical ventilation, Meta-analysis, Tracheostomy.

Contributions of each author:

Author 1 - Yun Ji.

Author 2 - Yumin Fang.

Author 3 - Libin Li.

Subgroup analysis: None. 